\title{
Hyalinizing Clear Cell Carcinoma of the Bronchus
}

\author{
Maryam Shahi $^{1} \oplus \cdot$ Michelle Dolan $^{1} \cdot$ Paari Murugan $^{1}$
}

Received: 2 February 2017 / Accepted: 4 May 2017 / Published online: 16 May 2017

(C) Springer Science+Business Media New York 2017

\begin{abstract}
Hyalinizing clear cell carcinoma (HCCC) is an uncommon low-grade minor salivary gland neoplasm that usually arises in the head and neck region. We report a 55-year-old man who presented with a $2.5 \mathrm{~cm}$ lung mass that was partially obstructing the right bronchus intermedius. The tumor consisted of cords and nests of clear and eosinophilic cells in a hyalinized stromal background. The neoplastic cells expressed cytokeratin (CK) 7, CK 5/6, high-molecular weight cytokeratin (34BE12), p63 and p40, while TTF-1, napsin A, CK20, S100, smooth muscle actin, synaptophysin and chromogranin were negative. Mucicarmine stain also was negative in the lesional cells. Fluorescence in situ hybridization using break apart probes revealed rearrangement of the Ewing Sarcoma Breakpoint Region 1 gene locus. The morphologic, immunophenotypic and cytogenetic findings confirmed the diagnosis of HCCC, most likely of bronchial submucosal gland origin. To our knowledge, only two other reports of primary pulmonary $\mathrm{HCCC}$ are available in English literature.
\end{abstract}

Keywords Hyalinizing clear cell carcinoma . Lung neoplasm $\cdot$ Salivary gland neoplasm $\cdot$ EWSR1 rearrangement

Maryam Shahi

shahi014@umn.edu

Michelle Dolan

dolan009@umn.edu

Paari Murugan

pmurugan@umn.edu

1 Department of Pathology and Laboratory Medicine, University of Minnesota, Minneapolis, MN 55455, USA

\section{Introduction}

Hyalinizing clear cell carcinoma (HCCC) was first described in 1994 as a distinctive low-grade carcinoma predominantly occurring in the intraoral minor salivary glands, more commonly in middle-aged women [1]. It is composed of cords, nests, islands and trabeculae of monomorphic glycogen rich clear and eosinophilic cells infiltrating a characteristic juxatapositioned hyalinized and fibrocellular stroma $[1,2]$.

In approximately $80 \%$ of patients, the primary tumor is located within the oral cavity, involving the minor salivary glands; the most frequent site of occurrence being the tongue and palate, which account for $>50 \%$ of tumors. Other less common sites include the parotid gland, subglottic larynx, nasopharynx, and hypopharynx [3]. Tumor metastasis has been reported in up to $25 \%$ of patients at initial presentation, primarily to regional lymph nodes and rarely to the lung [1,3-5]. Primary pulmonary HCCC is an extremely rare entity with only two previous reports $[6,7]$.

\section{Case Report}

A 55-year-old man with chronic obstructive pulmonary disease and history of smoking cessation 17 years earlier presented with complaints of back pain. Persistence pain prompted a chest CT scan that demonstrated a mass partially obstructing the right bronchus intermedius. Bronchoscopic examination showed luminal narrowing with a small knuckle of bulging tissue at the lateral wall and mostly unremarkable overlying mucosa (Fig. 1). The lesion was biopsied and a diagnosis of non-small cell carcinoma was rendered. A staging work-up showed no evidence of metastasis or other tumors, following which a video-assisted 
Fig. 1 Chest CT scan demonstrates a mass partially obstructing the right bronchus intermedius (a). Bronchoscopy shows an extrinsic narrowing within the bronchus intermedius with a small knuckle of bulging tissue (b)
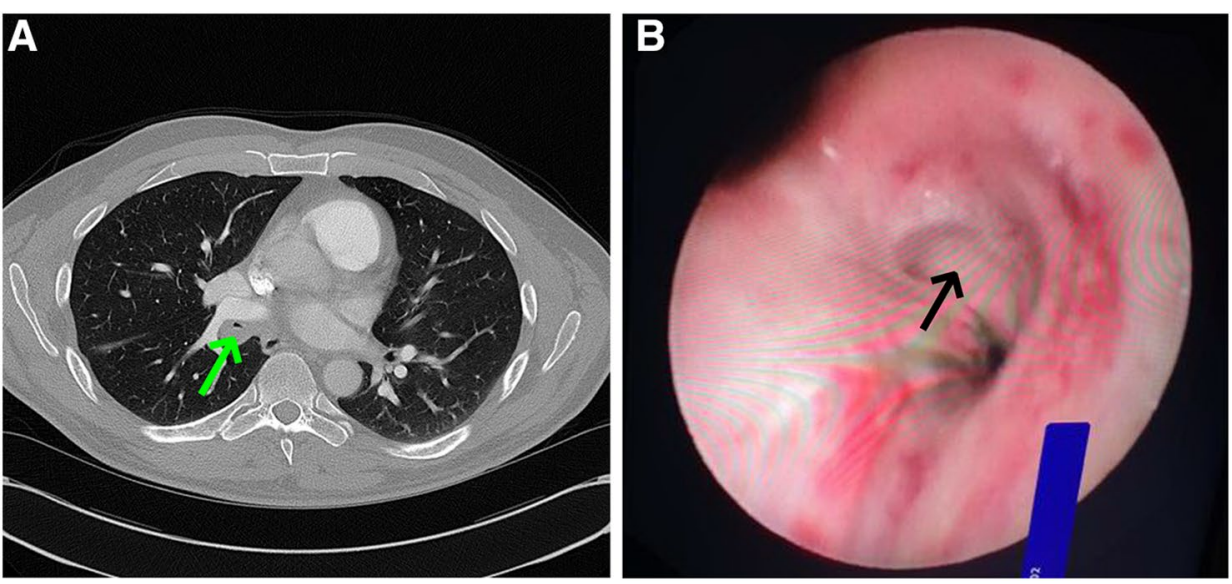

thoracoscopic bilobectomy (right middle and lower lobes) and mediastinal lymph node dissection was performed.

\section{Materials and Methods}

Immunohistochemistry - Slides were stained with antibodies against $\mathrm{CK} 5 / 6$, thyroid transcription factor-1(TTF-1) (mouse monoclonal, clone 8G7G3/1; Ventana), NapsinA (mouse monoclonal, clone TMU-Ad 02; Biocare), p40 (clone BC28; Biocare Medical), SMA (mouse monoclonal, clone 1A4; Ventana), p63 (mouse monoclonal, clone A4A; Ventana), CK 7 (clone OV-TL12/30; DAKO, Carpinteria, CA), CK 20 (clone S20.8; DAKO, Carpinteria, CA), S100 (rabbit polyclonal; Ventana), high-molecular weight cytokeratin (mouse monoclonal, clone 34 $\beta$ E12; DAKO, Carpinteria, CA), chromogranin (Mouse monoclonal, clone LK2H10; Ventana), and synaptophysin (rabbit monoclonal clone MRQ-40; Ventana). Mucicarmine staining was also performed.

Cytogenetics - FISH for EWSR1 (22q12) gene rearrangement was performed on formalin fixed paraffin embedded (FFPE) tumor sections according to standard protocol using a commercially available Ewing Sarcoma (EWS) break-apart probe (Abbott Molecular, Des Plaines, IL). Two hundred tumor nuclei were counted.

\section{Results}

The specimen consisted of a right middle and lower lobe resection that revealed a $2.5 \times 2.0 \times 0.9 \mathrm{~cm}$ yellow-tan firm grossly circumscribed submucosal lesion involving the right middle lobe bronchus intermedius. The adjacent alveolar parenchyma appeared uninvolved.

On hematoxylin and eosin-stained slides, a non-encapsulated, fairly circumscribed tumor with infiltrating borders was identified in the bronchial submucosa. Areas of permeation into the bronchial cartilage as well as foci of perineural invasion were noted. No lymphovascular invasion was identified. The lesion consisted of cords, trabeculae and nests of small to medium-sized cells in a background of alternating myxohyaline and cellular fibrous stroma.

The nuclei appeared fairly monotonous with open chromatin and inconspicuous nucleoli. The neoplastic cells possessed either clear or pale eosinophilic cytoplasm. Mitotic activity was minimal and necrosis was not identified. Foci of peritumoral lymphocytic infiltration were noted.

Scattered apparently entrapped bronchial glands and ducts were also noted (Fig. 2). 29 lymph nodes examined were free of tumor.

Immunohistochemical stains on the initial biopsy showed the neoplastic cells expressing p40, and CK5/6 while negative for synaptophysin and chromogranin. Immunostaining of the excised specimen showed diffuse positivity for CK7, high-molecular weight cytokeratin (34BE12) and p63. SMA highlighted the fibrocellular stroma but was negative in the tumor cells. CK20 and S100 were essentially negative in the tumor cells (Fig. 3). Mucicarmine stain also was negative in the lesional cells.

Of the 200 interphase cells examined, 55\% (110) had a signal pattern consistent with rearrangement of the EWSR1 locus (Fig. 4).

A subsequent PET scan failed to reveal additional tumor sites; the clinical, morphological, immunohistochemical and cytogenetic findings were consistent with a primary pulmonary hyalinizing clear cell carcinoma. The patient is recurrence free 20 months after diagnosis.

\section{Discussion}

In 1994, Milchgrub et al. initially described HCCC as a distinct entity that occurs predominantly in the intraoral minor salivary glands, more commonly in middle-aged women. 

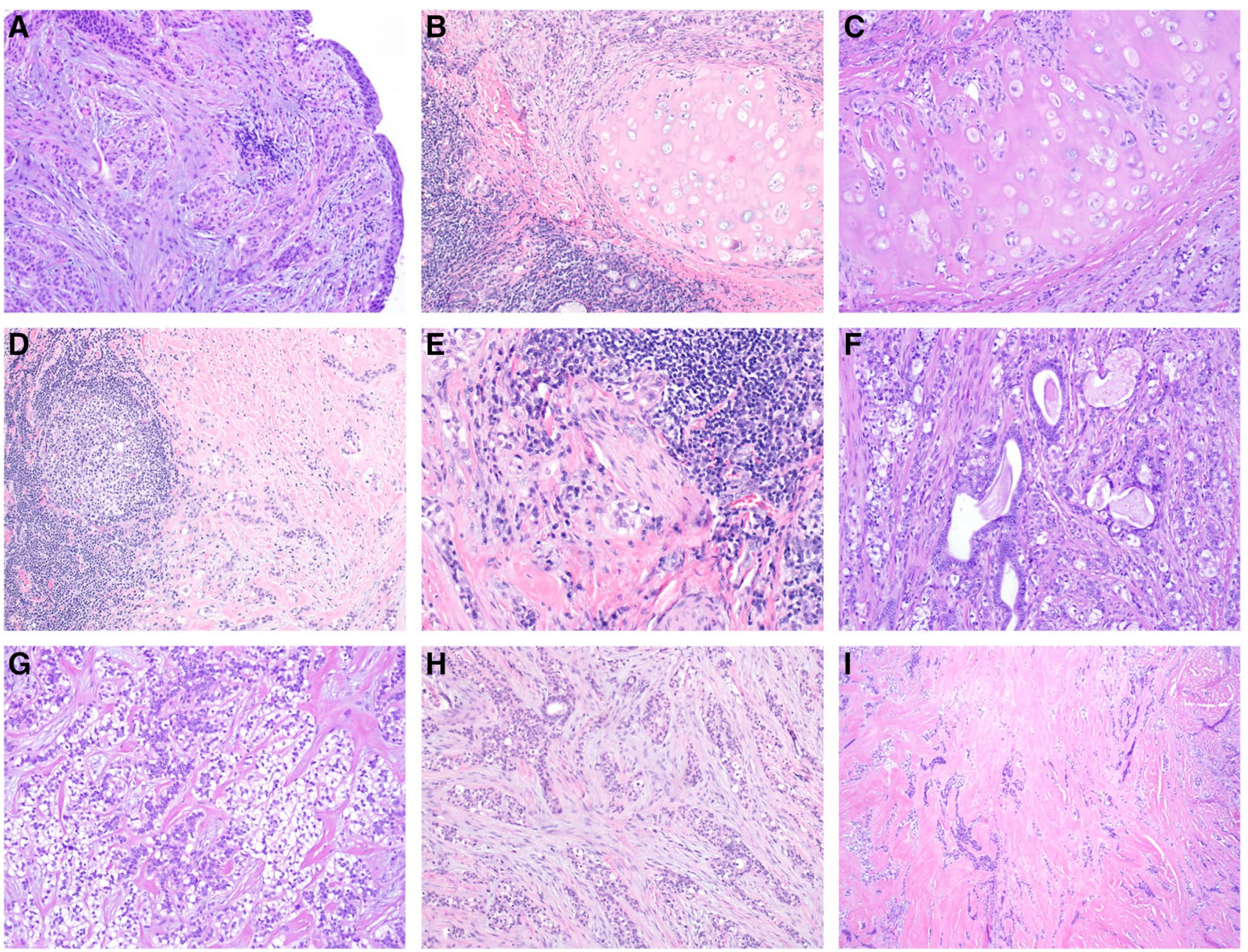

Fig. 2 The HCCC involves the overlying bronchial epithelium but no atypia or in-situ element is appreciated (a, $H \& E \times 100)$. Close proximity of the normal bronchial glands and the tumor is depicted (b, $H \& E \times 100)$. Tumor cells are seen infiltrating the bronchial cartilage (c, $H \& E \times 100)$. The lesion is fairly circumscribed, with peripheral

The most frequent sites of occurrence are the tongue and palate, accounting for $>50 \%$ of tumors. Other less common sites include parotid gland, subglottic larynx, nasopharynx, and hypopharynx [3]. Prior to this case, HCCC has only twice been previously reported as a primary lesion arising in the bronchial tree / lower respiratory tract $[6,7]$.

Hyalinizing clear cell carcinoma usually presents as a relatively circumscribed submucosal tumor that is unencapsulated and has a high tendency to insidiously infiltrate into the surrounding tissues, as seen in this case [1]. Microscopically, the tumor reveals various architectural patterns including cords, nests, islands and trabeculae of monomorphic glycogen rich clear cells and eosinophilic cells within a usually juxtaposed hyalinized and fibrocellular stroma [1, 2]. Rare scattered entrapped ducts and peritumoral lymphocytic infiltrate have also been reported [2,6]. Although the lymphoid aggregates $(\mathbf{d}, \mathrm{H} \& \mathrm{E} \times 100)$. Perineural invasion is readily identified $(\mathbf{e}, \mathrm{H} \& \mathrm{E} \times 200)$. Bronchial gland ducts appear entrapped within the tumor $(\mathbf{f}, \mathrm{H} \& \mathrm{E} \times 100)$. Tumor is composed of alternating eosinophilic and clear cells $(\mathbf{g}, \mathrm{H} \& \mathrm{E} \times 100)$ within a juxtaposed fibrocellular (h, H\&E×100) and hyalinizing stroma (i, H\&E×40)

common presentation is that of a painless mass, perineural invasion is almost invariably seen [1, 6-8].

Despite being classified by the WHO as an adenocarcinoma, the original description and subsequent reports have shown strong evidence of squamous differentiation in almost all of these tumors. This is underscored by occasional squamous pearl formation, p63 and $34 \mathrm{bE} 12$ positivity as well as the presence of desmosomes, glycogen, and tonofilaments on ultrastructural examination [1, 9, 10]. Mucinous differentiation may be focally seen in HCCC; rarely mucinous cells may be present in significant amounts to consider a diagnosis of mucin-depleted mucoepidermoid carcinoma (MEC) [1, 8]. In the current case, mucicarmine stain failed to demonstrate mucinous components.

Immunohistochemically, in keeping with the current case, HCCC demonstrates expression of p63, p40, 

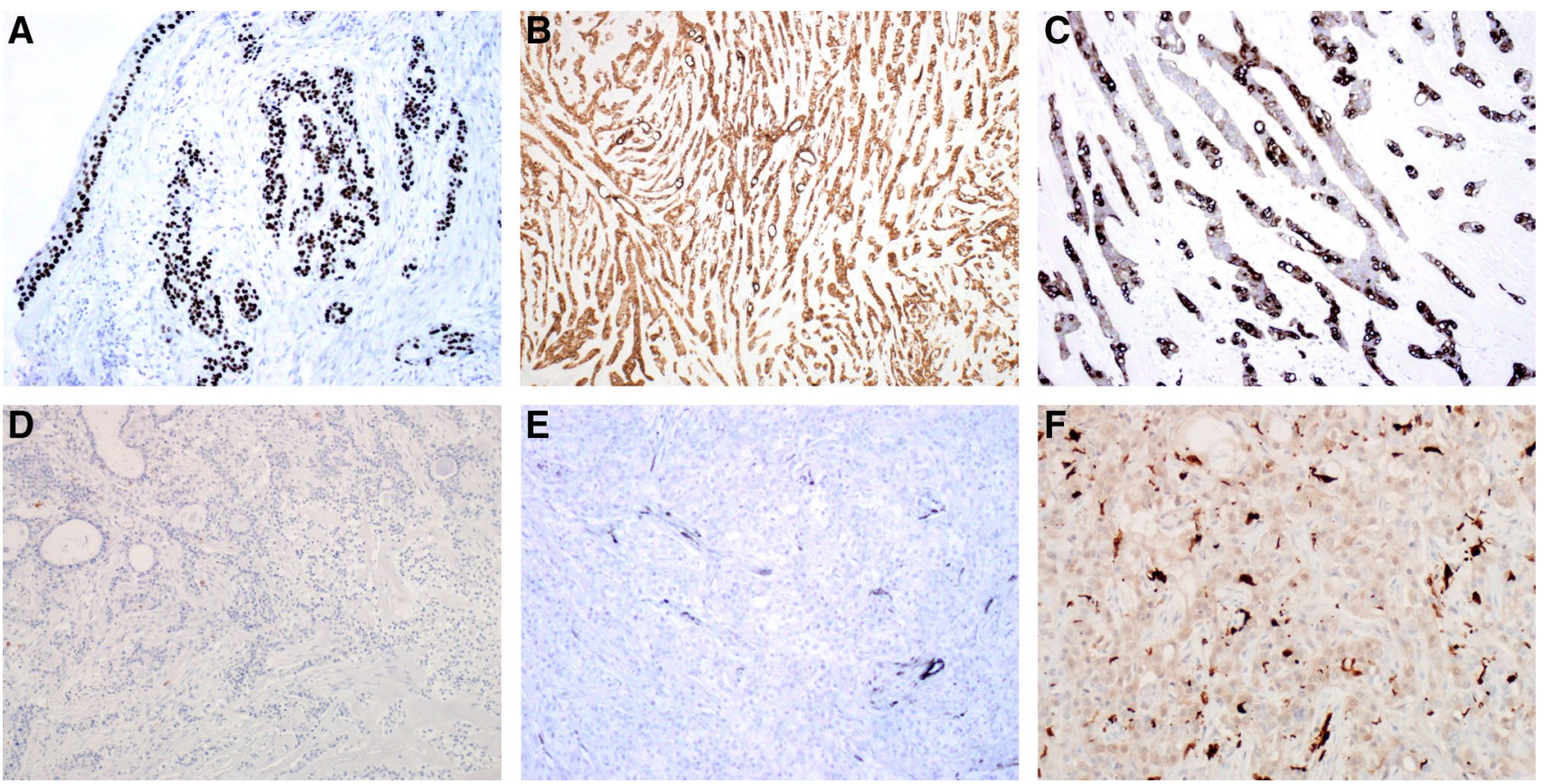

Fig. 3 The tumor cells diffusely express p40 (a, IHC $\times 40)$, highmolecular weight cytokeratin $(34 \mathrm{BE} 12)(\mathbf{b}, \mathrm{IHC} \times 40)$ and cytokeratin $7(\mathbf{c}, \mathrm{IHC} \times 100)$, while negative for cytokeratin $20(\mathbf{d}, \mathrm{IHC} \times 200)$, smooth muscle actin $(\mathbf{e}, \mathrm{IHC} \times 40)$, and $\mathrm{S} 100(\mathbf{f}, \mathrm{IHC} \times 200)$ excluding myoepithelial differentiation

Fig. 4 Fluorescence in-situ hybridization with EWSR1 break apart probe; the fused yellow signal is considered normal and once rearrangement of the EWSR1 gene happens, the break-apart probe shows one separate green and red signal per cell. $1 \mathrm{R} 1 \mathrm{G} 1 \mathrm{~F}: 1$ red 1 green 1 fused
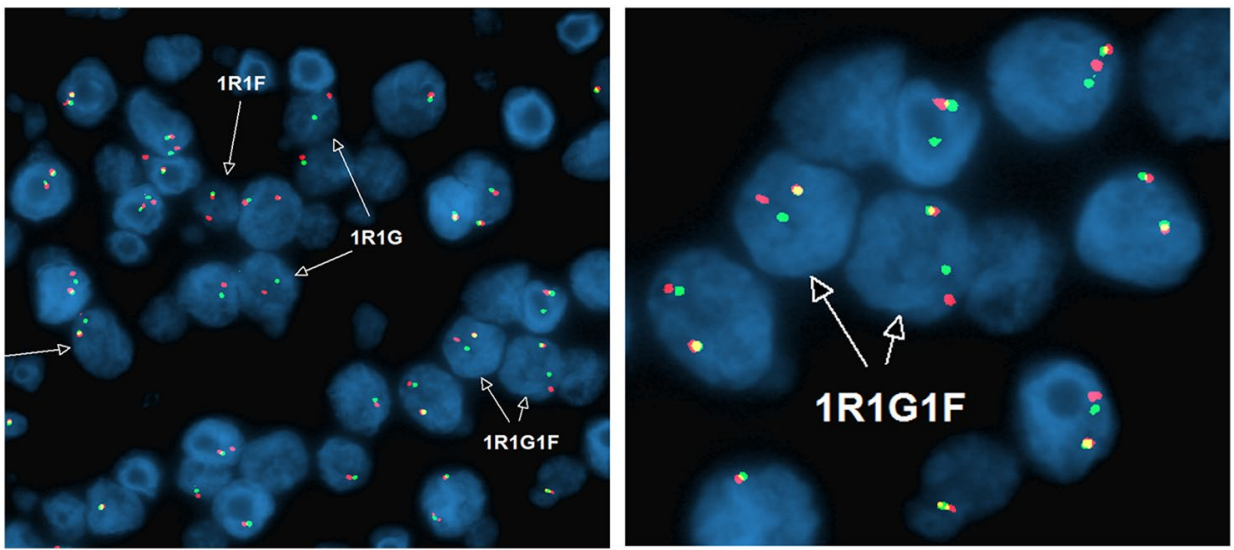

cytokeratin 5/6 and cytokeratin $7[1-3,8,9,11]$. Positivity for EMA, CAM5.2, CK 19 and CK 14 has been observed; however, S100 protein, SMA, muscle specific actin and calponin, the myoepithelial differentiation markers, are negative [2].

Antonescu et al. identified an in-frame fusion between EWSR1 exon 11 and ATF1 exon 3 as a novel recurrent genetic abnormality in $85-92 \%$ of HCCC while examination of the major mimickers failed to show EWSR1-ATF1 rearrangement $[6,12-14]$. The only exception was clear cell odontogenic carcinoma, which shares both the morphologic and gene rearrangement in a majority of cases, thus considered to be the odontogenic counterpart of
HCCC [12, 13]. While rearrangement of EWSR1 gene is seen in several neoplasms, including Ewing sarcoma, desmoplastic small round cell tumor, clear cell sarcoma and myxoid chondrosarcoma, these tumors can be easily ruled out based on other clinicopathologic features.

The potential differential diagnoses for HCCC based on its histological features include squamous cell carcinoma, clear cell oncocytoma, MEC, epithelial-myoepithelial carcinoma, clear cell myoepithelioma, myoepithelial carcinoma, acinic cell carcinoma, and metastatic renal cell carcinoma [1-6]. Excluding squamous carcinoma may be particularly challenging in the lung, especially on biopsy material given the presence of epithelioid cells positive for 
CK5/6 and p63 embedded in a fibrosclerotic stroma. These markers also react with myoepithelial tumors and MEC. The absence of myoepithelial marker expression (e.g. S100 and SMA) excludes the tumors with myoepithelial differentiation, including epithelial-myoepithelial carcinoma, clear cell myoepithelioma and myoepithelial carcinoma. As previously mentioned, although intracytoplasmic mucin may rarely be seen in HCCC, features characteristic of MEC such as cyst formation, solid growth of squamoid and intermediate cells are not found in the former. In challenging cases, cytogenetic studies would be revealing, as MEC show MAML2 gene rearrangement and almost never EWSR1 [1, 8, 15]. Given the peculiar location in the current case primary pulmonary tumors were also considered. The epithelioid cytomorphology and occasional cribriform pattern, along with positive CK7 immunostaining raised the possibility of adenocarcinoma while the organoid architecture and bronchial submucosal location suggested a well differentiated neuroendocrine tumor. Negative immunohistochemistry for TTF-1, Napsin A, synaptophysin and chromogranin ruled out these possibilities.

Hyalinizing clear cell carcinoma is a low-grade carcinoma with excellent prognosis despite local invasion and locoregional metastasis [1, 3, 4]. Although a rare aggressive HCCC with widespread metastasis has been reported distant metastasis is not a common manifestation [16]. In light of the fact that only four cases have been reported so far, it remains to be seen if pulmonary HCCC demonstrates clinical behavior similar to its head and neck counterpart. The current patient is alive without disease (AWD) 20 months after surgery while the other three were AWD at 10,17 and 17 months at the time of the reports $[6,7]$.

\section{Conclusion}

In conclusion, we report an extremely rare case of cytogenetically proven primary pulmonary hyalinizing clear cell carcinoma. Awareness of the tumor arising in this location in conjunction with the characteristic morphologic appearance and ancillary studies should help make the correct diagnosis and guide appropriate conservative surgery.

\section{References}

1. Milchgrub S, Gnepp DR, Vuitch F, et al. Hyalinizing clear cell carcinoma of salivary gland. Am J Surg Pathol. 1994;18:74-82.
2. Weinreb I. Hyalinizing clear cell carcinoma of salivary gland: a review and update. Head and Neck Pathol. 2013;7:20-9.

3. Solar AA, Schmidt BL, Jordan RC. Hyalinizing clear cell carcinoma: case series and comprehensive review of the literature. Cancer. 2009;115:75-83.

4. O'Sullivan-Mejia ED, Massey HD, Faquin WC, et al. Hyalinizing clear cell carcinoma: report of eight cases and a review of literature. Head Neck Pathol. 2009;3(3):179-85.

5. Ereño C, Grande J, Alija V, Yarnoz J, Bilbao FJ. Hyalinizing clear cell carcinoma of the hypopharynx metastasizing to the lung: a case report. Histopathology. 2000;37:89-91.

6. García J, Jin L, Jackson S, et al. Primary pulmonary hyalinizing clear cell carcinoma of bronchial submucosal gland origin. Human Pathol. 2015;46:471-5.

7. Shah AA, Mehrad M, Kelting SM, et al. An uncommon primary lung tumour: hyalinizing clear cell carcinoma, salivary glandtype. Histopathology. 2015;67(2):274-6.

8. Antonescu CR, Katabi N, Zhang L, et al. EWSR1-ATF1 fusion is a novel and consistent finding in hyalinizing clear-cell carcinoma of salivary gland. Genes Chromosomes Cancer. 2011;50:559-70.

9. Dardick I, Leong I. Clear cell carcinoma: review of its histomorphogenesis and classification as a squamous cell lesion. Oral Surg Oral Med Oral Pathol Oral Radiol Endod. 2009;108:399-405.

10. Ellis GL, Auclair PL. Armed forces Institute of pathology (AFIP) atlas of tumor pathology. In: Tumors of the salivary glands (fourth series, fascicle 9). Silver Spring: APR Press; 2008. p. 301-9.

11. Tanguay J, Weinreb I. What the EWSR1-ATF1 fusion has taught us about hyalinizing clear cell carcinoma. Head Neck Pathol. 2013;7:28-34

12. Bilodeau EA, Hoschar AP, Barnes EL, et al. Clear cell carcinoma and clear cell odontogenic carcinoma: a comparative clinicopathologic and immunohistochemical study. Head Neck Pathol. 2011;5:101-7.

13. Bilodeau EA, Weinreb I, Antonescu CR, et al. Clear cell odontogenic carcinomas show EWSR1 rearrangements: a novel finding and a biological link to salivary clear cell carcinomas. Am J Surg Pathol. 2013;37:1001-5.

14. Shah AA, LeGallo RD, van Zante A, et al. EWSR1 genetic rearrangements in salivary gland tumors: a specific and very common feature of hyalinizing clear cell carcinoma. Am J Surg Pathol. 2013;37:571-8.

15. Roden AC, Garcia JJ, Wehrs RN, et al. Histopathologic, immunophenotypic and cytogenetic features of pulmonary mucoepidermoid carcinoma. Mod Pathol. 2014;27:1479-88.

16. O'Regan E, Shandilya M, Gnepp DR, et al. Hyalinizing clear cell carcinoma of salivary gland: an aggressive variant. Oral Oncol. 2004:40:348-52. 